\title{
Management of Vascular Malformations in Dangerous Area of Oral Cavity: The Iranian Experience and Review of Treatment Modalities
}

\author{
Somayeh Alirezaei ${ }^{1}, M^{2}$ aryam Baharvand ${ }^{2}$, Masood Rezaei ${ }^{1}$, Arash Azizi ${ }^{1}$, \\ Bita Tavakoli ${ }^{*}$ \\ ${ }^{1}$ Dental School, Islamic Azad University, Tehran, Iran \\ ${ }^{2}$ Dental School, Shahid Beheshti University of Medical Sciences, Tehran, Iran \\ ${ }^{3}$ Oral and Maxillofacial Medicine Department, Dental School, Islamic Azad University, Tehran, Iran \\ Email: ${ }^{\text {bitatavakoli2014@gmail.com }}$
}

Received 18 January 2014; revised 23 February 2014; accepted 3 March 2014

Copyright (C) 2014 by authors and Scientific Research Publishing Inc.

This work is licensed under the Creative Commons Attribution International License (CC BY).

http://creativecommons.org/licenses/by/4.0/

\section{(c) (i) Open Access}

\begin{abstract}
Aim: To evaluate the effect of a highly potent corticosteroid (dexamethasone) in the treatment of vascular malformation when the location is difficult to reach and complications such as uncontrolled bleeding is predictable in surgery. Background: Vascular malformation is not a common lesion in oral cavity especially in alveolar ridge with extension to the pillar uvula. These lesions arise from capillary or venous malformations with various surgical or non-surgical treatment modalities. Case Description: We performed weekly intralesional injection of dexamethasone in a patient with a vascular malformation in alveolar ridge extending to the lingual side of alveolar ridge and posterior extension to the uvula. Complete resolution of lesion was observed after 6th injection. Conclusion: Intralesional injection of dexamethasone is a potentially curative method to treat oral vascular malformation. Clinical Significance: Injection of dexamethasone is a simple and cost-effective therapy that can be used as a safe treatment for vascular malformations prior to or as a substitute for surgery.
\end{abstract}

\section{Keywords}

Vascular Malformation; Intralesional Injection; Dexamethasone

\footnotetext{
${ }^{*}$ Corresponding author.
}

How to cite this paper: Alirezaei, S., et al. (2014) Management of Vascular Malformations in Dangerous Area of Oral Cavity: The Iranian Experience and Review of Treatment Modalities. Open Journal of Stomatology, 4, 115-120. 


\section{Case Description}

A 30-year-old male presented to the Department of Oral and Maxillofacial Medicine complaining of an oral lesion which had developed 4 months before his attendance to our department. The lesion was red/bluish red in color, measuring $3 \times 5 \mathrm{~cm}$ in the first visit (Figure 1) and reducing in size on consecutive visits (Figures 1-6). It had pebbly surface. The lesion was prone to bleeding spontaneously or by minor trauma, therefore, the diagnosis of a vascular malformation was made clinically.

The vascular malformation was located on the alveolar ridge extending to the lingual side and posteriorly to the uvula, making surgery difficult because of the fear of massive bleeding.

We decided to try dexamethasone injection for the patient. An informed written consent was obtained from the patient, and our study was approved by Medical Ethics Committee of Shahid Beheshti University of Medical Sciences.

We injected $1 \mathrm{ml}$ of $8 \mathrm{mg} / \mathrm{ml}$ dexamethasone by insulin needles into the base of lesions to prevent any leakage and causing less discomfort and pain. No local anesthesia was required. The patient received 6 consecutive shots with one-week intervals, and the lesion was examined on days 7, 14, 21, 28, 35, and 42 to evaluate the response (Figures 1-6).

The patient was followed for 18 months, and is still symptom free.

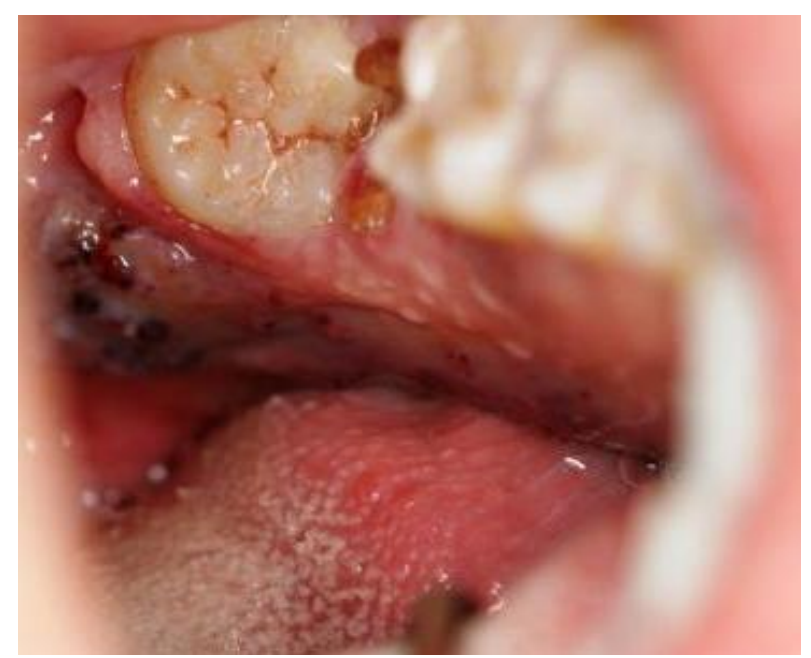

Figure 1. First visit.

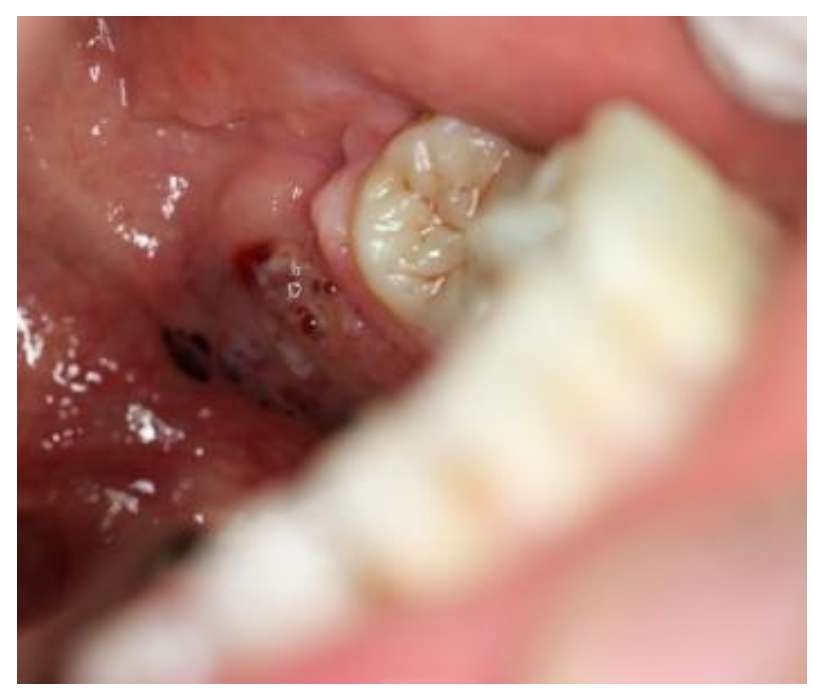

Figure 2. Second visit. 


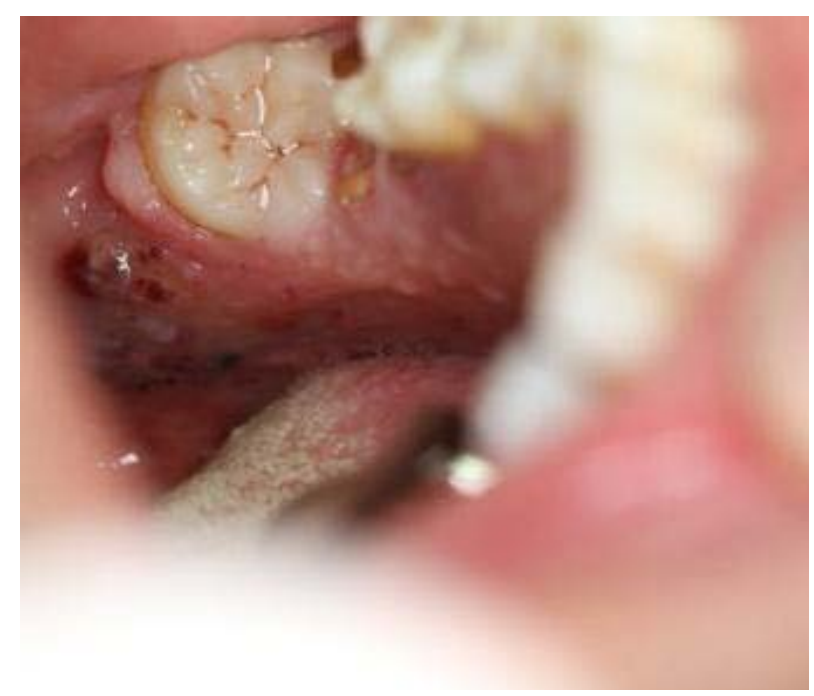

Figure 3. Third visit.

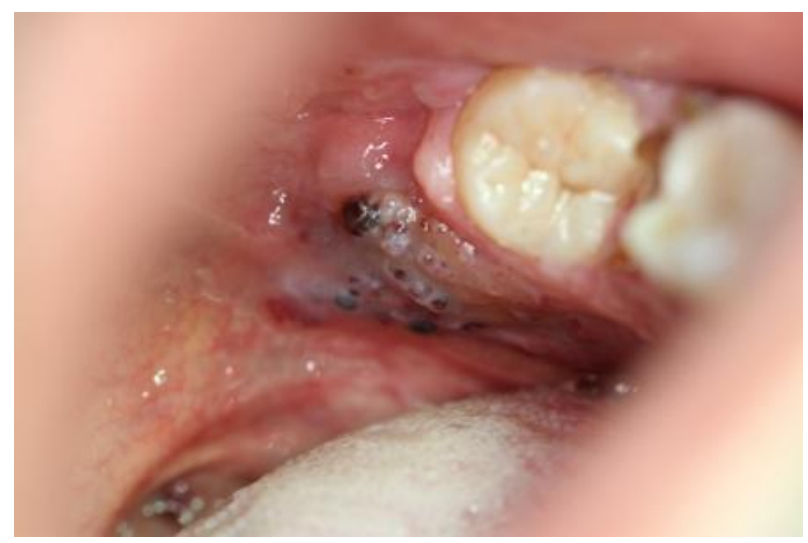

Figure 4. Fourth visit.

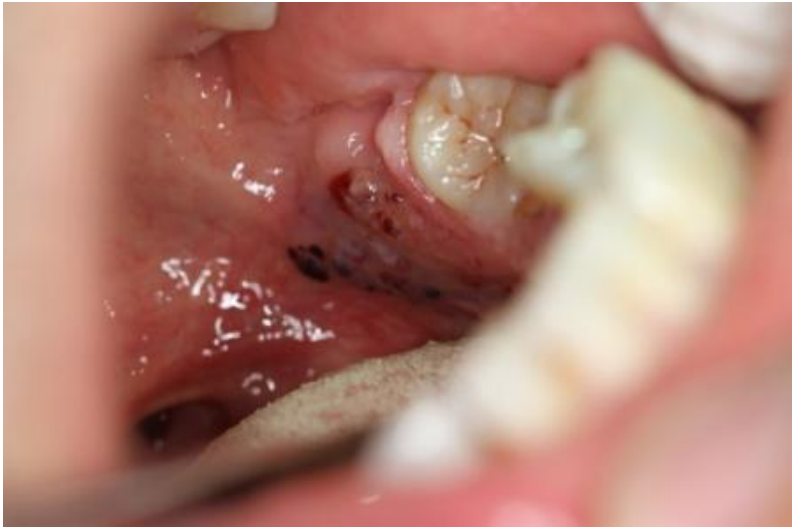

Figure 5. Fifth visit.

\section{Discussion}

In order to evaluate the effectiveness and probable complications of intralesional dexamethasone, this study was performed on a patient with vascular malformation. We used a highly potent corticosteroid, dexamethasone, because results and complications of surgery were unpredictable. 


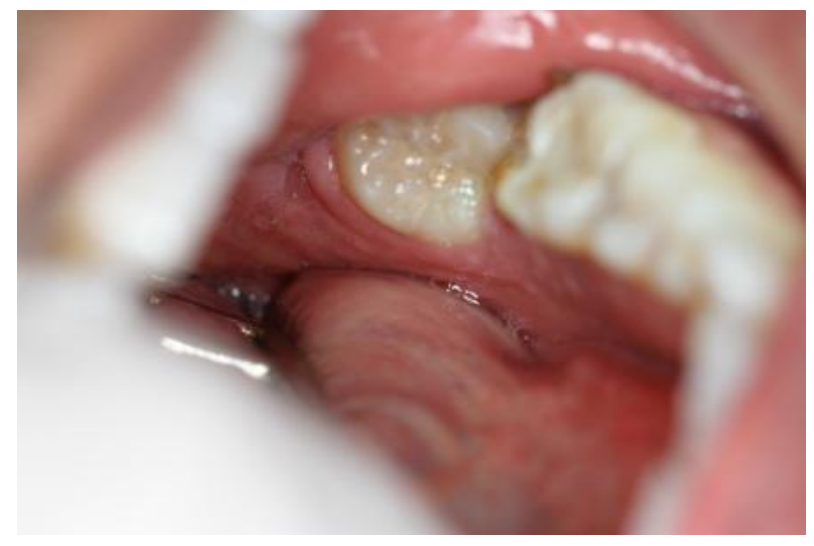

Figure 6. Sixth visit.

The treatment had minimal pain and no local anesthesia was required. The time of procedure was short, making it comfortable for the patient (important in children).

Regarding the cost, dexamethasone injection therapy is more cost effective than surgery, cryotherapy or laser ablation, and is available in almost every medical or dental setting.

To best of our knowledge this is the first report to show that vascular malformations can be treated by the use of dexamethasone.

\section{Review of Literature}

Vascular lesions are common congenital abnormalities with a reported incidence of $10 \%-12 \%$. Approximately $60 \%$ of cases occur in the head and neck region [1]. The majority of experts believe that many cases (more than $50 \%$ ) of hemangioma in the past have been misdiagnosed and mistreated because the term hemangioma has been used to represent a multitude of vascular entities. In order to make the best treatment decision for the patient, knowing the exact diagnosis as well as the differential diagnosis of vascular anomalies is extremely important. Treatment must be tailored based on the understanding of the pathogenesis of the disease process [1]. Mulliken and Glowacki have developed a biological classification of vascular anomalies including physical findings, clinical behavior and cellular kinetics [2]. Based on that classification, hemangioma and vascular malformations are two different entities; with different natural course and sometimes entirely opposite prognosis.

Usually, the hemangiomas that grow rapidly at the beginning have a self-limiting growth and undergo substantial remission before the child reaches the age of five to seven years. Therefore, treatment is usually necessary if the hemangioma threatens the function of an organ; for example impairing the vision, breathing, eating or hearing, or in case of development of local or systemic complications such as bleeding, infection or necrosis. Otherwise, no treatment is usually needed [1] [3].

In contrast to hemangioma, vascular malformations almost never undergo remission and continue to grow in proportion to the body growth. Unlike hemangiomas, they seldom have a fast growth unless in the case of acute bleeding of presence of arteriovenous shunting. Vascular malformations can be further categorized based on their predominant blood vessel: arterial, venous, capillary, lymphatic or mixed. Mulliken and Glowacki classification has led to a modification of the original classification. For example because port-wine stains are in fact composed of postcapillary venules, the term venular malformation is more appropriate term to describe it [2]. Waner and Suen in their study have classified vascular malformations: venular, venous, lymphatic and arteriovenous [4]. The first step in management should be correct recognition of the exact diagnosis: hemangiomas or vascular malformations. Correct differential diagnosis is critical for appropriate treatment as for example; steroids are effective for proliferating hemangiomas, but non-effective for involuted hemangiomas and vascular malformations.

Vascular malformations almost never involute, therefore early treatment is indicated in their management. Treatment depends on several factors including anatomical location, type, depth and the extent of the lesion, and among several methods of treatment, sclera therapy and laser treatment followed by surgical excision are the most popular ones [5]-[7]. For more extensive lesions, a multidisciplinary approach is preferred. 
In addition to histological classifications, vascular malformations are also categorized based on their hemodynamic features during angiography: "Low-flow" lesions include venular, venous and lymphatic malformations, and "high-flow" lesions include arterial, arterio venous malformations and arteriovenous fistulas [3].

\section{Treatment Modalities}

Surgery: Indications of surgery in the involuted phase include: damaged skin, abnormal contour (fibro fatty residuum), distortion or destruction of an important anatomic feature, and need for staged resection or reconstruction. In the surgery, care must be taken to preserve as much normal structures as possible in order to achieve the best cosmetic results [4].

Laser therapy: The type and extent of treatment for a venous malformation depends on the type, location and depth of the lesion. Superficial venous malformations such as these located in the oral mucosa can be treated with a Nd:YAG laser. Superficial components of compound venous malformations can also be treated with Nd:YAG laser since the vessels that make up the lesion venous malformation are quite large. Size of the vessel is also a determining factor for choosing the method of treatment. Flash lamp pumped dye laser (pulse dye laser, tunable dye laser) is suitable for small vessels. For intermediate vessels, a laser with a longer exposure time (copper vapor or KTP laser) is a reasonable option. In case of very large vessels, a continuous wave Nd:YAG laser is often needed.

In compound lesions, at first superficial component should be treated with a laser, then the deep component should be treated with surgical resection or sclerotherapy. If treated with laser, usually more than one therapeutic sessions (6 - 8 weeks apart) are required to achieve complete involution of the lesion. Laser surgery should be done before surgical removal in order to preserve enough skin for reconstruction.

Surgical removal of the deep component should be done 6 to 8 weeks after the laser surgery, so that the swelling caused by laser therapy settles down. One strategy to reduce postoperative swelling is peri-operative administration of steroids.

This is essential in laser therapy of some anatomical locations such as orbit, oral cavity and the airway. Complications after laser therapy include hypo-pigmentation hyper-pigmentation, and scarring [8].

Laser treatment is effective only on superficial lesions (less than $3 \mathrm{~mm}$ ). In case of presence of a lesion with a deep component such as lesions located in parotid gland, surgery is the preferred initial treatment. After the surgery when the lesion is well exposed, treatment with Nd:YAG laser can be performed [6] [7]. In case of good response to irradiation, further resection is not needed, however otherwise, the irradiated lesion can be surgically removed or injected with a sclerosant agent such as bleomycin [7] [8].

Sclerotherapy: Sclerotherapy consists of direct injection of a sclerosing agent into the lesion. Sclerosing agents cause destruction of endothelial surfaces and thrombosis, which results in fibrosis and obliteration of the vessel lumen [9]. For small and middle-sized superficial and deep venous malformations of the maxillofacial and cervical regions, sclerotherapy can be considered the treatment of choice. Several sclerosing agents are available, however, none of them can be considered optimal for this purpose in terms of safety and efficacy. Choice of sclerosing agent depends mainly on physician's preference, size and location of the lesions, and draining veins within the lesions [8] [10].

Several studies have reported that in order to occlude thin peripheral arteries and veins, absolute ethanol is the most effective substance [10]-[12]. However, ethanol injection is painful, and in some cases can result in extensive tissue necrosis in small and superficial venous malformations or in lesions with low draining speed veins. It may also cause nerve damage in lesions that are in close proximity to major nerves [10] [13]. For these reasons, ethanol sclerotherapy is better to be reserved for the treatment of larger and deeper malformations, or lesions with high draining speed veins documented on direct venography. It can also be used in combination with bleomycin for giant lesions. Bleomycin is less potent than ethanol, but it is associated with much less risk of ulceration and nerve damage, and it is also associated with lower incidence of side effects and allergic reactions [7] [8].

To perform sclerotherapy, intraluminal position of the needle tip can also be confirmed by aspiration of blood; however, it can also be performed more accurately under the guidance of color duplex or MRI.

In general, confirmation by aspiration of blood can be considered safe for small superficial lesions. For deeper and larger venous malformations, injection must be monitored with an imaging modality to prevent accidental intra-arterial injection. 
The most common local complication of sclerotherapy is marked swelling, especially after injection of ethanol. This complication is the consequence of extravasation of the sclerosing agent into the skin capillaries [14]. Extravasation causes intra-lesional thrombus and edema which can be reduced by administration of short-term, low-dose corticosteroids.

When this side effect occurs, the skin begins to blanch, and in these cases, the procedure should be discontinued immediately. Other systemic complications are rare, such as allergic reactions, cardiac arrest, pulmonary embolism, neurologic deficit, or deep vein thrombosis [12]. Sclerotherapy of venous malformations is often followed by frequent recanalization of the lesions, in which treatment can be repeated. In resistant cases, surgical resection can be performed [12].

\section{Conclusion}

Injection of dexamethasone is a simple, repeatable, cost effective and potentially curative method of treatment, and can be used as the first choice or substitute for surgery in the treatment of vascular malformations.

\section{Acknowledgements}

The authors are greatly thankful to Mr. Fathi (the patient) for with being on-time and fully cooperative, as well as the Medical Ethics Committee of Shahid Beheshti University of Medical Sciences which approved the study.

\section{References}

[1] Finn, M.C., Glowacki, J. and Mulliken, J.B. (1983) Congenital Vascular Lesions: Clinical Application of a New Classification. Journal of Pediatric Surgery, 18, 894-900. http://dx.doi.org/10.1016/S0022-3468(83)80043-8

[2] Mulliken, J.B. and Glowacki, J. (1982) Hemangiomas and Vascular Malformation in Infants and Children: A Classification Based upon Endothelia Characteristics. Plastic and Reconstructive Surgery, 69, 412-422. http://dx.doi.org/10.1097/00006534-198203000-00002

[3] Jackson, I.T., Carreno, R., Potparic, Z. and Hussain, K. (1993) Vascular Malformations, and Lymphovenous Malformations: Classification and Methods of Treatment. Plastic and Reconstructive Surgery, 91, 1216-1230. http://dx.doi.org/10.1097/00006534-199306000-00006

[4] Waner, M. and Suen, J.Y. (1999) Hemangiomas and Vascular Malformations of the Head and Neck. Wiley-Liss, New York, 1-12.

[5] Pence, B., Aybey, B. and Ergenekon, G. (2005) Outcomes of $532 \mathrm{~nm}$ Frequency-Doubled Nd:YAG Laser Use in the Treatment of Port-Wine Stains. Dermatologic Surgery, 31, 509-517. http://dx.doi.org/10.1111/j.1524-4725.2005.31152

[6] Poetke, M., Urban, P., Philipp, C. and Berlien, H.P. (2005) Hemangiomas-Classification, Diagnostic and Laser Treatment. Przegląd Flebologiczny, 13, 77-84.

[7] Mathur, N.N., Rana, I., Bothra, R., Dhawan, R., Kathuria, G. and Pradhan, T. (2005) Bleomycinsclerotherapy in Congenital Lymphatic and Vascular Malformations of Head and Neck. International Journal of Pediatric Otorhinolaryngology, 69, 75-80. http://dx.doi.org/10.1016/j.ijporl.2004.08.008

[8] Muir, T., Kirsten, M., Fourie, P., Dippenaar, N. and Ionescu, G.O. (2004) Intralesionalbleomycin Injection (IBI) Treatment for Haemangiomas and Congenital Vascular Malformations. Pediatric Surgery International, 19, 766-773. http://dx.doi.org/10.1007/s00383-003-1058-6

[9] Yamaki, T., Nozaki, M. and Sasaki, K. (2000) Color Duplex-Guided Sclerotherapy for the Treatment of Venous Malformations. Dermatologic Surgery, 26, 323-328. http://dx.doi.org/10.1046/j.1524-4725.2000.99248.x

[10] Rimon, U., Garniek, A., Galili, Y., Golan, G., Bensaid, P. and Morgan, B. (2004) Ethanol Sclerotherapy of Peripheral Venous Malformations. European Journal of Radiology, 52, 283-287. http://dx.doi.org/10.1016/j.ejrad.2003.09.010

[11] Shireman, P.K., McCarthy, W.J., Yao, J.S. and Vogelzang, R.L. (1997) Treatment of Venous Malformations by Direct Injection with Ethanol. Journal of Vascular Surgery, 26, 838-844. http://dx.doi.org/10.1016/S0741-5214(97)70098-3

[12] Yakes, W.F., Haas, D.K., Parker, S.H., et al. (1989) Symptomatic Vascular Malformations: Ethanol Embolotherapy. Radiology, 170, 1059-1066.

[13] Berenguer, B., Burrows, P.E., Zurakowski, D. and Mulliken, J.B. (1999) Sclerotherapy of Craniofacial Venous Malformations: Complications and Results. Plastic and Reconstructive Surgery, 104, 12-15. http://dx.doi.org/10.1097/00006534-199907000-00003

[14] de Lorimier, A.A. (1995) Sclerotherapy for Venous Malformations. Journal of Pediatric Surgery, 30, 188-193. http://dx.doi.org/10.1016/0022-3468(95)90558-8 